\title{
MEJORANDO EL ACCESO A LA ATENCIÓN DEL ABORTO TERAPÉUTICO EN EL INSTITUTO NACIONAL MATERNO PERINATAL EN SALVAGUARDA DEL DERECHO A LA VIDA Y LA SALUD DE LA MUJER
}

\author{
IMPROVING ACCESS TO THERAPEUTIC ABORTION CARE AT THE \\ NATIONAL MATERNAL PERINATAL INSTITUTE IN SAFEGUARDING THE \\ RIGHT TO LIFE AND HEALTH OF WOMEN
}

\author{
Jacqueline Lourdes Chapa Romero ${ }^{1, a}$
}

\begin{abstract}
RESUMEN
Objetivo. Describir las acciones que realiza el Instituto Nacional Materno Perinatal, respecto al acceso a los servicios de salud para la atención de la mujer para aborto terapéutico. Materiales y Métodos. Estudio, descriptivo, transversal. Se revisaron todos los expedientes de solicitudes de aborto terapéutico y que fueron atendidos en el Instituto Nacional Materno Perinatal durante los periodos de enero a diciembre del 2019 y 2020. Resultados. Se atendieron 117 y 59 solicitudes para aborto terapéutico durante los periodos 2019 y 2020 respectivamente. La edad no es impedimento para solicitar el procedimiento de interrupción terapéutica voluntaria del embarazo, solo en los casos de menores de edad, se acompaña la firma de los padres o apoderados de la menor. Además, la institución cumple con informar al Ministerio Público para garantizar las medidas legales de tutela jurisdiccional en defensa de la libertad sexual de la menor. Todas las solicitudes, han seguido el procedimiento administrativo para su evaluación en junta médica, en los cuales se determina la aceptación o la negación del procedimiento. En 2019 y 2020 fueron aprobadas en junta médica 105 y 55 solicitudes respectivamente; y denegadas 12 y 4 casos respectivamente. Cumplieron con los criterios de las guías nacionales del MINSA en 90 y 46 solicitudes durante los años 2019 y 2020 respectivamente; quedando en alcance del protocolo médico institucional en 27 y 13 casos respectivamente. Cuentan con informe de salud mental aceptada en junta médica en 105 y 55 casos durante los periodos 2019 y 2020 respectivamente. Conclusiones. Todas las solicitudes de interrupción terapéutica voluntaria del embarazo, presentadas por las gestantes, han seguido el procedimiento administrativo para su evaluación mediante junta médica, sin embargo, en algunos expedientes no se han encontrado copia del informe de salud mental.
\end{abstract}

Palabras clave: Aborto terapéutico; Acceso a la atención; Salvaguarda del derecho a la vida; Salud de la mujer. (Fuente: DeCS BIREME).

\begin{abstract}
Objective. Describe the actions carried out by the National Maternal Perinatal Institute, regarding access to health services for the care of women for therapeutic abortion. Materials and Methods: Descriptive, cross-sectional study. All the files of requests for therapeutic abortion that were attended at the National Maternal-Perinatal Institute during the periods from January to December 2019 and 2020 were reviewed. Results. 117 and 59 requests for therapeutic abortion were attended during the 2019 and 2020 periods respectively. Age is not an impediment to request the procedure of voluntary therapeutic interruption of pregnancy, only in the cases of minors, the signature of the minor's parents or guardians is accompanied. In addition, the institution complies with informing the Public Ministry to guarantee the legal measures of judicial protection in defense of the sexual freedom of the minor. All applications have followed the administrative procedure for evaluation by the medical board, in which the acceptance or denial of the procedure is determined. In 2019 and 2020, 105 and 55 applications were approved by the medical board, respectively; and denied 12 and 4 cases respectively. They met the criteria of the MINSA national guidelines in 90 and 46 requests during the years 2019 and 2020 respectively; remaining within the scope of the institutional medical protocol in 27 and 13 cases respectively. They have a mental health report accepted by the medical board in 105 and 55 cases during the 2019 and 2020 periods respectively. Conclusions. All the requests for voluntary therapeutic interruption of pregnancy, presented by the pregnant women, have followed the administrative procedure for their evaluation by a medical board, however, in some files, no copy of the mental health report has been found.
\end{abstract}

Keywords: Therapeutic abortion; Access to care; Safeguarding the right to life; Women's health. (Source: MeSH NLM)

\footnotetext{
a Abogada. Asistente Profesional I. Jefa de Unidad Funcional de Secretaría General de la Dirección General. Estudiante de Post Grado en la Facultad de Derecho de la Universidad Nacional Mayor de San Marcos. Estudios de Especialización en Derecho Administrativo en la Universidad Continental.
}

ID Código ORCID: https://orcid.org/0000-0003-3221-7792, Jacqueline Lourdes Chapa Romero

Citar como: Chapa Romero JL. Mejorando el acceso a la atención del aborto terapéutico en el Instituto Nacional Materno Perinatal en salvaguarda del derecho a la vida y la salud de la mujer. Rev Peru Investig Matern Perinat 2021; 10(3): 30-37 DOI https://doi.org/10.33421/inmp.2021248 


\section{INTRODUCCIÓN}

La Organización Mundial de la Salud, como organismo internacional especializado en gestionar políticas de prevención, promoción e intervención mundial en salud integral, establece que el aborto seguro, aborto legal o llamado en el Perú el aborto terapéutico, debe garantizar entre otros, la atención de los establecimientos de salud en condiciones de bioseguridad, infraestructura, tecnología y recursos humanos disponibles para brindar atención oportuna y necesaria para el manejo de estos casos.

En el Perú el aborto terapéutico se encuentra exceptuado de sanción penal, regulado en el artículo $119^{\circ}$ del Código Penal que textualmente señala "No es punible el aborto practicado por un médico con el consentimiento de la mujer embarazada o de su representante legal, si lo tuviere, cuando es el único medio para salvar la vida de la gestante o para evitar en su salud un mal grave y permanente".

El Ministerio de Salud ${ }^{1}$ emitió la Resolución Ministerial N486-2014/MINSA mediante el cual aprobó la "Guía Técnica Nacional para la estandarización del procedimiento de la atención integral de la gestante en la interrupción voluntaria por indicación terapéutica del embarazo menor de 22 semanas con consentimiento informado en el marco de lo dispuesto en el artículo $119^{\circ}$ del código penal"; sin embargo, actualmente la cobertura de este servicio de salud no están siendo brindado con efectividad a la población.

Actualmente el Instituto Nacional Materno Perinatal de Lima, es el hospital que brinda el mayor número de atenciones y viene cumpliendo lo establecido en la guía técnica, por su condición de establecimiento de nivel III-2 de mayor complejidad en salud sexual y salud reproductiva, en donde se viene brindando una atención de calidad para estos casos, lo que nos lleva a considerar que este modelo de atención debe ser socializado en los establecimientos de II y III de nivel de atención.

No obstante, estando casi a 7 años de la implementación de dicha guía técnica, la casuística ha permitido observar aspectos éticos y legales en la evaluación de los casos, lo que conlleva a evitar quejas, reclamos, o denuncias, por la negativa en la atención de las gestantes que acuden al Instituto Nacional Materno Perinatal, orientando las actuaciones basadas en principios éticos en defensa del derecho a la vida y la salud de la gestante.

El objetivo de la presente investigación es describir las acciones que realiza el Instituto Nacional Materno Perinatal, respecto al acceso a los servicios de salud para la atención de la mujer para aborto terapéutico.

\section{AVANCES EN LA IMPLEMENTACIÓN DE LA GUÍA TÉCNICA NACIONAL SOBRE EL ABORTO TERAPEUTICO}

El Estado protege la vida ${ }^{2}$, la salud ${ }^{3}$ y la autonomía de las personas, en concordancia a los derechos de primera y segunda generación. La Guía técnica tiene como objetivo estandarizar los procedimientos para la atención integral de la gestante en los casos de interrupción voluntaria por indicación terapéutica del embarazo menor de veintidós (22) semanas con consentimiento informado, cuando es el único medio para salvar la vida de la gestante o para evitar en su salud un mal grave y permanente, en concordancia al artículo $119^{\circ}$ del Código Penal ${ }^{4}$. Esta atención se realizará en los establecimientos de salud con categoría del segundo nivel a más de atención del sistema de salud a nivel nacional, para ello se debe garantizar la disponibilidad de recursos humanos, infraestructura equipamiento, medicamentos e insumos mínimos.

Desde la aprobación de la norma técnica nacional en el año 2014, ha opinión de los expertos profesionales de la salud que conforman la Sociedad Peruana de Obstetricia y Ginecología (SPOG), han manifestado que existen dificultades administrativas en los establecimientos de salud, para brindar la atención del aborto terapéutico e interrumpir el embarazo por las causales establecidas, en otros existen dificultades éticas y legales para brindar dicha atención, siendo que en alguno de los casos los médicos gineco-obstetras no comprenden que el concepto del aborto terapéutico, consiste en un procedimiento que toma en cuenta los principios de la bioética, y otro supuesto es que el personal de la salud aún no conoce las bases legales para la atención del aborto terapéutico que están incluidas en la Ley N${ }^{\circ} 26842$ Ley General de Salud ${ }^{5}$, la Ley $N^{\circ} 29414$ Ley que establece los derechos de las personas usuarias ${ }^{6}$, la Declaración Universal de los Derechos Humanos y la Convención sobre la eliminación de todas las formas de discriminación contra la mujer.

En la actualidad el manejo de los procedimientos de interrupción terapéutica del embarazo, se vienen realizando en los establecimientos de salud asignados por el Ministerio de Salud, siendo que la mayoría de casos atendidos han sido realizados en la ciudad de Lima. Es por ello, que consideramos importante revisar los casos, basados en la experiencia del Instituto Nacional Materno Perinatal, por su categoría de mayor nivel III-2 en el sector salud y que atiende la mayor demanda en atención obstétrica. 


\section{CONSIDERACIONES PARA LA PROTECCIÓN LEGAL DE LOS SUJETOS QUE INTERVIENEN EN EL PROCEDIMIENTO MÉDICO.}

Consideramos que desde el año la aprobación de la guía técnica nacional, corresponde a la sociedad civil y científica en salud, apoyar que las medidas que emita el estado contribuyan a la mejora de la calidad de atención a los usuarios. En tanto, se han emitido algunas investigaciones a razón del seguimiento y cumplimiento de la norma técnica nacional. Uno de los artículos de investigación titulado "Barreras para el Aborto Seguro en el marco de la ley peruana”, publicado en la revista de la Sociedad Peruana de Obstetricia y Ginecología, reportado por Távara $L^{7}$ propuso ciertas intervenciones para lograr el objetivo de la guía técnica nacional, entre los cuales debo destacar en primer orden el "promover la información y educación oportuna a las usuarias, empoderándolas en su derecho a la interrupción legal del embarazo por razones médicas" . La participación de las organizaciones de la sociedad civil es clave en esta intervención. Otro aspecto a destacar, está referido al "fortalecimiento oportuno a los proveedores de los servicios de salud, en temas de bioética y clarificación de valores en la prestación del aborto terapéutico". En esta misma línea de acción sugiere "remover algunas restricciones de carácter administrativo para facilitar el acceso al aborto terapéutico". En este contexto, encontramos como actores sociales privados y públicos, los cuales se menciona a continuación:

Actores sociales privados más importantes:

Sociedad Peruana de Obstetricia y Ginecología Centro de Promoción y Defensa de Derechos Reproductivos - PROMSEX

Centro de la mujer peruana Flora Tristan

\section{Actores sociales públicos más importantes:}

Defensoría del Pueblo

Ministerio de Salud

Ministerio de la Mujer y Poblaciones Vulnerables

Dado que es un tema que implica la participación multidisciplinaria de diversos actores no solo en temas de salud, sino de legalidad y defensa de los derechos humanos con enfoque en salud pública, por tanto, la toma de decisiones en favor de las mujeres, debe estar conformado por profesionales médicos en ginecología y obstetricia, psiquiatría, además de profesionales en derecho, que permitan formular propuestas de intervención que favorezcan a los partícipes en el procedimiento del aborto terapéutico, según los aspectos que se detallan en el la tabla 1.

Tabla 1. Consideraciones que deben tomar en cuenta la paciente gestante y el profesional médico. Bien jurídico protegido: La vida y la salud de la mujer

CONSIDERACIONES QUE DEBEN TOMAR EN CUENTA LA PACIENTE GESTANTE Y EL PROFESIONAL MÉDICO

Bien Jurídico Protegido: La vida y la salud de la mujer

\begin{tabular}{|c|c|c|}
\hline Sujetos & $\begin{array}{l}\text { Inseguridad de ser sometidos } \\
\text { a denuncia por: }\end{array}$ & $\begin{array}{l}\text { La actuación no amerita sanción, } \\
\text { de acuerdo a: }\end{array}$ \\
\hline $\begin{array}{l}\text { 1. Mujer gestante } \\
\text { 2. El Médico } \\
\text { (Especialista en Ginecología } \\
\text { y Obstetricia) }\end{array}$ & $\begin{array}{l}\text { Delito de Aborto - Código penal } \\
\text { Código Penal } \\
\text { Art. } 114^{\circ} \text { Autoaborto } \\
\text { Art. } 115^{\circ} \text { Aborto consentido } \\
\text { Art. } 116^{\circ} \text { Aborto sin consentimiento } \\
\text { Art. } 118^{\circ} \text { Aborto preintencional } \\
\text { Art. } 120^{\circ} \text { Aborto sentimental y eugenésico. }\end{array}$ & $\begin{array}{l}\text { Art. } 119^{\circ} \text { Aborto Terapéutico - } \\
\text { Código Penal } \\
\text { (A partir de las } 12 \text { semanas hasta } \\
\text { menos de } 22 \text { semanas de embarazo) } \\
\text { Art. } 20^{\circ} \text { Exención de responsabilidad } \\
\text { penal (...) } \\
\text { 8) El que obra por disposición de } \\
\text { la ley, en cumplimiento de un deber } \\
\text { o en el ejercicio legítimo de un } \\
\text { derecho, oficio o cargo. (...) } \\
\text { 10) El que actúa con el consentimiento } \\
\text { válido del titular de un bien jurídico } \\
\text { de libre disposición. }\end{array}$ \\
\hline $\begin{array}{l}\text { 3. El médico especialista } \\
\text { (Especialista en Ginecología } \\
\text { y Obstetricia) }\end{array}$ & $\begin{array}{l}\text { Procedimiento Disciplinario Administrativo } \\
\text { en el Sector Público. } \\
\text { Art. } 85 \text { Ley } 30057 \text { Ley Servicio Civil } \\
\text { b) La reiterada resistencia al cumplimiento } \\
\text { de las órdenes de sus superiores } \\
\text { relacionadas con sus labores. } \\
\text { n) La negligencia en el desempeño de las } \\
\text { funciones }\end{array}$ & $\begin{array}{l}\text { Colegio Médico del Perú. Código de Ética } \\
\text { y Deontología } \\
\text { Artículo } 53^{\circ} \text { El médico no debe interrumpir } \\
\text { la asistencia de un paciente que le ha } \\
\text { sido confiado (...) } \\
\text { Artículo } 55^{\circ} \text { En pacientes que requiere } \\
\text { procedimientos diagnósticos o terapéuticos } \\
\text { que impliquen riesgos mayores que el } \\
\text { mínimo, el médico debe solicitar consentimiento } \\
\text { informado por escrito, por medio del cual se les } \\
\text { comunique en qué consisten, así como las } \\
\text { alternativas posibles, la probable duración, } \\
\text { los límites de confidencialidad, la relación } \\
\text { beneficio/riesgoy beneficio/costos. }\end{array}$ \\
\hline
\end{tabular}




\section{PROCEDIMIENTO ADMINISTRATIVO (TRÁMITES) PARA EL ACCESO AL ABORTO SEGURO}

La interrupción del embarazo, es una cuestión ética, que según opinión de la Federación Internacional de Ginecología y Obstetricia (FIGO), señala que la legislación del aborto traduce una ruptura en la lógica y la práctica política y social, en relación a lo conservador de la moral que confina a las mujeres en el único papel de madres y esposas, degradando a la maternidad porque la entiende como destino biológico y no como elección ética y cuestión de derecho.

Como se puede advertir, la figura del aborto, es un tema muy sensible para la sociedad, en nuestro caso nos remitimos a la excepción del aborto terapéutico, ya que excluye de responsabilidad a la gestante y al médico. Para el manejo de estos casos, en el Perú contamos con la guía técnica nacional, emitida conforme a nuestro ordenamiento legal, ya que reglamenta el supuesto de hecho contenido en el artículo $119^{\circ}$ del código penal, que forma parte de nuestro sistema jurídico vigente y despliega todos sus efectos jurídicos en todo ámbito nacional, de modo que goza del principio de legalidad y se ajusta a nuestra constitución.

En tanto la guía técnica nacional, ha previsto la presentación de solicitudes en la vía administrativa por las mujeres gestantes, por lo que la revisión de estos expedientes, nos podría permitir conocer la implementación de la guía técnica nacional durante los años 2019 y 2020.

La presente investigación, ha revisado y evaluado las solicitudes de las mujeres gestantes, desde un enfoque jurídico propio del derecho, en donde se analiza la licitud de la interrupción voluntaria por indicación terapéutica del embarazo en los casos de 22 semanas o más de gestación, por las causales que señala la guía de procedimientos que viene implementando el Instituto Nacional Materno Perinatal, considerando que la negación o el retraso para el acceso al aborto seguro y a la atención posterior al aborto, son formas de violencia de género que al incrementar la exposición de la mujeres a estresores aumentan el riesgo de depresión, que es una enfermedad discapacitante y con riesgo de muerte prematura por suicidio. Además, que el retraso de la atención para la interrupción terapéutica del embarazo puede implicar también un mayor riesgo con efectos en las complicaciones físicas graves para la gestante que pueden conllevar incluso a la muerte. Todas estas situaciones pueden llegar a configurar torturas o tratos crueles, inhumanos o degradantes. Asimismo, negar o no implementar servicios de salud que solo las mujeres requieren, constituye una expresión de discriminación.

\section{PROPUESTAS DE MEJORA EN LOS PROCEDIMIENTOS ADMINISTRATIVOS $Y$ ASISTENCIALES EN EL INSTITUTO NACIONAL MATERNO PERINATAL}

La guía técnica nacional del Ministerio de Salud, tiene como objetivo estandarizar los procedimientos para la atención de la gestante, por lo que el Instituto Nacional Materno Perinatal, logró actualizar en sus documentos de gestión el aborto terapéutico, en una guía interna de procedimiento aprobada con resolución directoral en el año 2018. A razón de ello, me he permitido formular un cuadro comparativo en el cual se muestra la incorporación de procedimientos administrativos - asistenciales, resguardando los aspectos éticos y legales que garanticen la oportuna atención de las pacientes.

\begin{tabular}{|c|c|}
\hline Guía Técnica MINSA & Guía Procedimientos INMP \\
\hline $\begin{array}{l}\text { Aprobado con Resolución Ministerial } \\
\qquad N^{\circ} 486-2014-\text { MINSA }^{1}\end{array}$ & $\begin{array}{l}\text { Aprobado con Resolución Directoral } \\
\qquad N^{\circ} 155-2018-D G-I N M P^{9}\end{array}$ \\
\hline $\begin{array}{l}\text { Objetivo: estandarizar los procedimiento para la atención } \\
\text { integral de la gestante en los casos de interrupción } \\
\text { voluntaria del embarazo. }\end{array}$ & $\begin{array}{l}\text { Objetivo: plantear una herramienta moderna y práctica a } \\
\text { fin de brindar una atención segura y de calidad a la } \\
\text { gestante. }\end{array}$ \\
\hline $\begin{array}{l}\text { Procedimientos Administrativos Asistenciales } \\
\text { 1. El médico tratante advierte que el embarazo poneen } \\
\text { riesgo la vida o la salud de la gestante. } \\
\text { 2. A petición de la gestante, presenta solicitud del caso } \\
\text { a la Jefatura del Departamento de Gineco Obstetricia } \\
\text { con conocimiento a Dirección General. } \\
\text { 3. La jefatura del departamento, constituye y convoca } \\
\text { Junta Médica. } \\
\text { 4. El médico tratante informará la decisión. } \\
\text { 5. (...) }\end{array}$ & $\begin{array}{l}\text { Procedimientos Administrativos Asistenciales: } \\
\text { 1. El médico tratante informa los diagnósticos que } \\
\text { ponen en riesgo la vida o la salud de la gestante. } \\
\text { 2. La gestante será evaluada por el Servicio de Psiquia- } \\
\text { tría. } \\
\text { 3. La gestante o su representante legal, presenta su } \\
\text { solicitud dirigido al Director General } \\
\text { 4. La Dirección Ejecutiva de Ginecología y Obstetricia, } \\
\text { refiere al Departamento de Gineco Obstetricia, para } \\
\text { que el Servicio se haga cargo del proceso. } \\
\text { 5. En un plazo de } 48 \text { horas se realiza junta médica. } \\
\text { 6. (...) }\end{array}$ \\
\hline
\end{tabular}


Una de las cuestiones más importantes en los procedimientos de mejora y de calidad de atención al paciente que ha implementado el Instituto Nacional Materno Perinatal, ha sido lo relacionado a la evaluación preliminar de la paciente en salud mental, lo cual no se ha considerado en el procedimiento del MINSA, pero su informe ayuda a la toma de decisiones del profesional médico gineco obstetra, ya que los sentimientos o pensamientos de las personas, no se puede tratar con medicamento o cirugías, sino mediante un análisis profundo de un especialista. Por ello en una investigación realizada por Rondón $\mathrm{M}^{10}$ titulada "Salud Mental y Aborto Terapéutico", describe: "La salud es un bien preciado; el derecho de todas las personas al nivel más alto de salud disponible está consagrado en documentos internacionales y en la Constitución peruana. La salud mental es inherente al concepto de bienestar integral, y resulta indispensable para la vida digna y el desarrollo individual y de las comunidades. El embarazo no deseado supone una situación de estrés, ya que amenaza el proyecto de vida de la mujer y la enfrenta al riesgo de pobreza, violencia y discriminación. Por ello, produce manifestaciones depresivas y ansiosas con un sustrato neurobiológico que pueden llevar a la mujer a la depresión posparto. La depresión es una enfermedad del cerebro que produce incapacidad, tiene un curso recidivante y conlleva riesgo de suicidio".

Esto indica que el factor de salud mental debería ser valorado como un instrumento primordial para la evaluación de las solicitudes de pacientes gestantes que acuden a un establecimiento de salud para solicitar la interrupción voluntaria terapéutica de su embarazo, quienes han sido informadas previamente por un profesional médico especialista del estado y los riesgos que implica para su vida y salud.

\section{RESULTADOS DE LA REVISIÓN DE LAS SOLICITUDES PRESENTADAS DURANTE EL PERIODO 2019 Y 2020}

El Instituto Nacional Materno Perinatal, ha recibido durante el año 2019 un total de 117 solicitudes de gestantes, acogiéndose a lo señalado en la Guía Técnica del INMP9, para acceder al aborto terapéutico como un medio legal tanto para la mujer como para el médico en donde se realizaría el procedimiento, lo cual se realiza previa a una junta médica que tiene en cuenta las causales que ponen en riesgo la salud o la vida de la gestante. En el año 2020, recibieron 59 solicitudes, que en razón a la pandemia del COVID-19 hubo un descenso, ya que hubo restricciones a nivel nacional para la atención de pacientes en consulta externa, lo es cual es necesario, puesto en el control prenatal se puede detectar estos casos y de acuerdo al diagnóstico médico la paciente puede iniciar un trámite administrativo para la evaluación de su caso en junta médica.

Se ha considerado, evaluar tres aspectos:

1. Rango de edad de las pacientes que lo solicitan.

2. Los expedientes cuentan con copia de la Junta Médica aceptadas y denegadas.

3. Los expedientes cuentan con copia de la Junta Médica según semana de gestación.

4. Los expedientes cuentan con informe de salud mental por semana de gestación.

Figura 1. Rango de edad de las pacientes que lo solicitan interrupción terapéutica de embarazo.
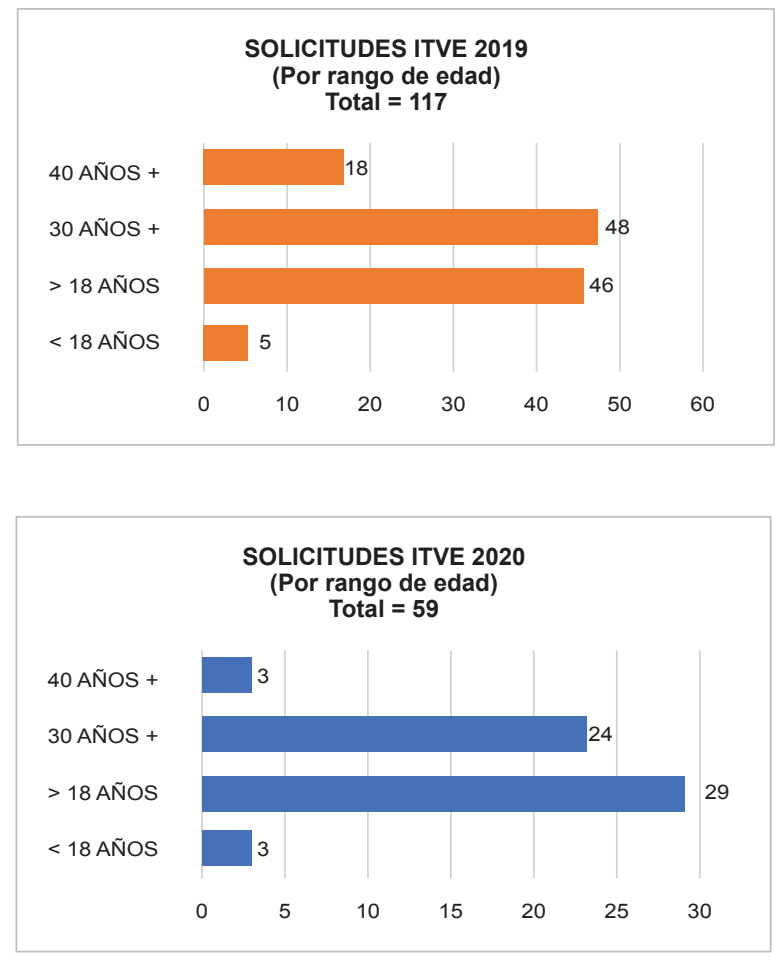

Fuente: Datos obtenidos de los expedientes administrativos que obran en los archivos de la dirección general del INMP (2021).

Se puede observar que el rango de edad no es impedimento para solicitar el procedimiento de interrupción terapéutica voluntaria del embarazo, solo en los casos de menores de edad, se acompaña la firma de los padres o apoderados de la menor. Además, la institución cumple con informar al Ministerio Público para garantizar las medidas legales de tutela jurisdiccional en defensa de la libertad sexual de la menor. 
Figura 2. Los expedientes cuentan con copia de la Junta Médica aceptadas y denegadas, periodos 2019 y 2020
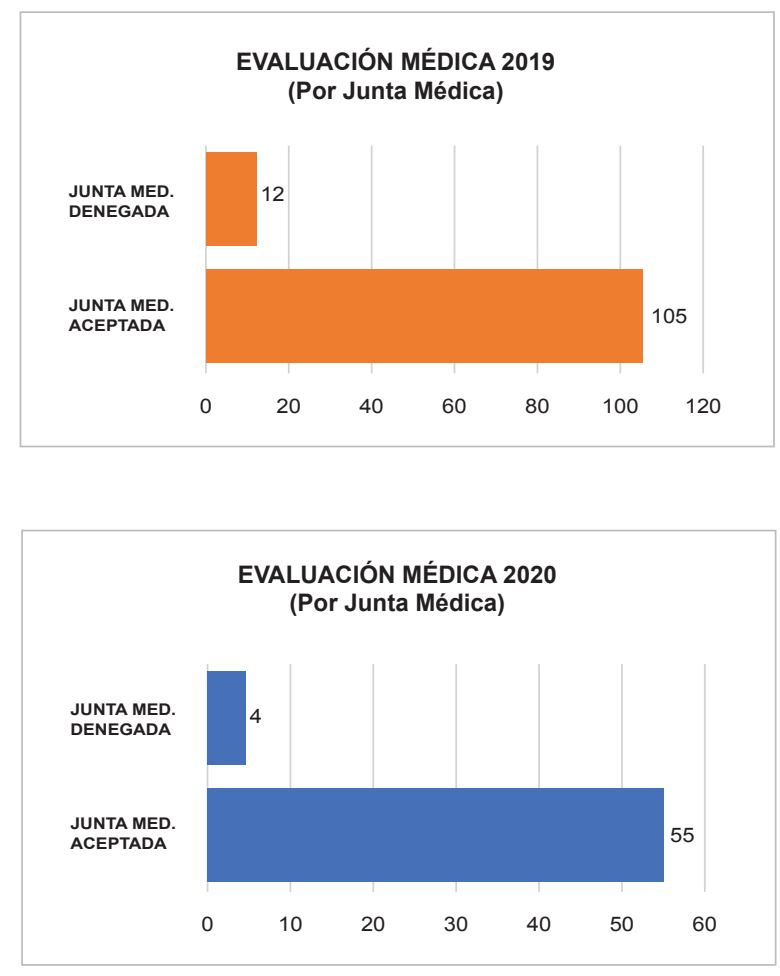

Fuente: Datos obtenidos de los expedientes administrativos que obran en los archivos de la dirección general del INMP (2021).

Se puede observar que todas las solicitudes de interrupción terapéutica voluntaria del embarazo, han seguido el procedimiento administrativo para su evaluación en junta médica, en los cuales se determina la aceptación o la negación del procedimiento. Aquellos casos denegados, fueron porque no estaban ceñidos en las causales clínicas ni en las obstétricas, contempladas en los protocolos médicos, en amparado a los instrumentos de orden normativo.

Figura 3. Los expedientes cuentan con copia de Junta Médica según semanas de gestación, periodo 2019

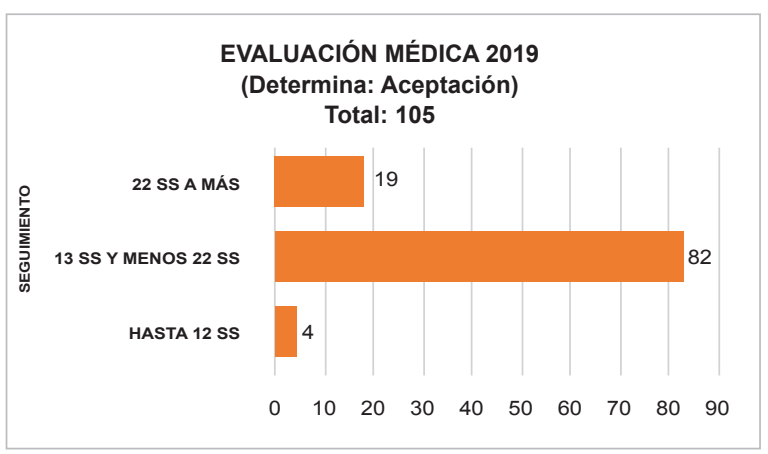

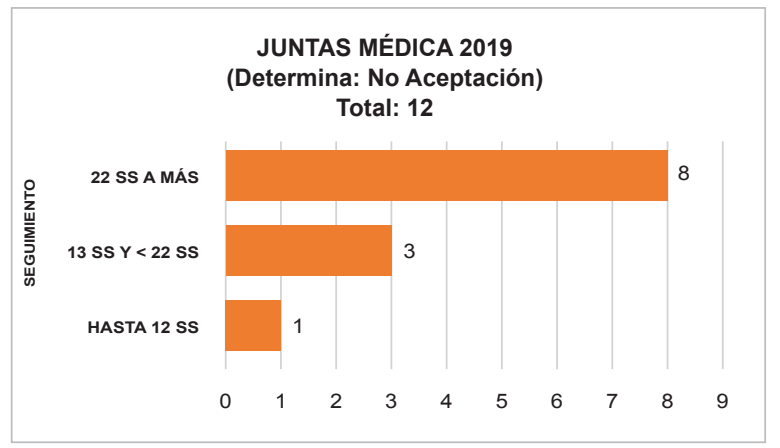

Fuente: Datos obtenidos de los expedientes administrativos que obran en los archivos de la dirección general del INMP (2021)

En el año 2019, de los 117 casos, se ha revisado las solicitudes de pacientes según las semanas de gestación, ya que la guía nacional del MINSA, precisa los procedimientos de menos de 22 semanas, siendo un total de 90 solicitudes que cumplen dicho requisito, por lo tanto 27 solicitudes quedan en alcance del protocolo médico en obstetricia y ginecología del INMP.

Figura 4. Los expedientes contienen informe de salud mental discutidas en Junta Médica, periodo 2019.
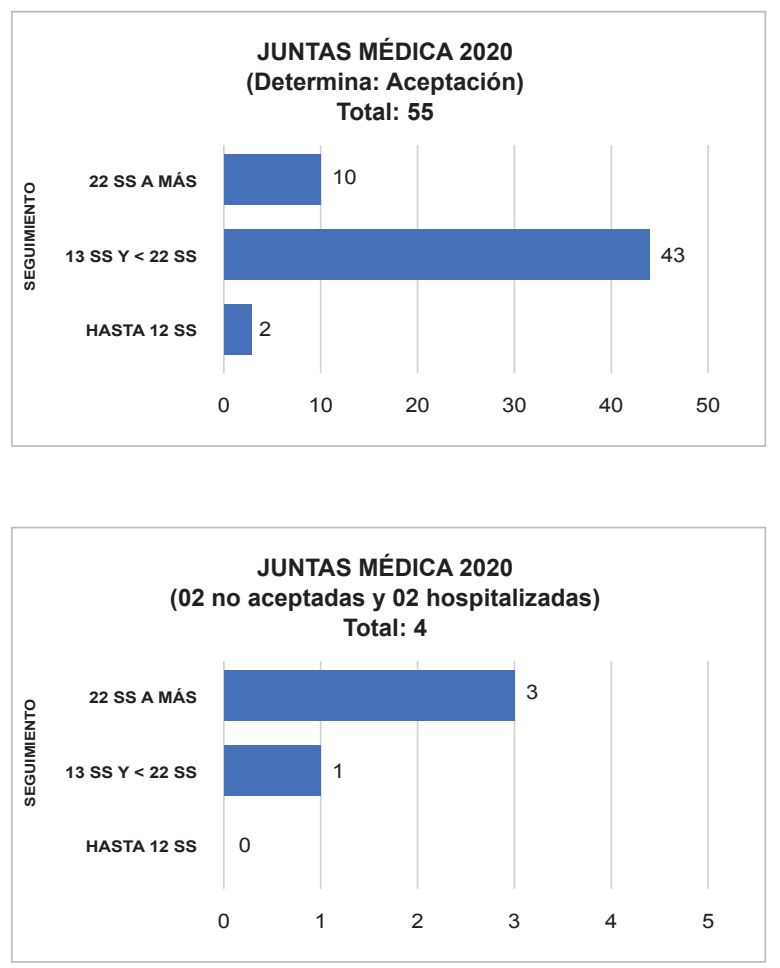

Fuente: Datos obtenidos de los expedientes administrativos que obran en los archivos de la dirección general del INMP (2021).

En el año 2020, de los 59 casos, se ha revisado las solicitudes de pacientes según las semanas de gestación, ya que la guía nacional del MINSA, precisa los procedimientos de menos de 22 semanas, siendo un total de 46 solicitudes que cumplen los requisitos del MINSA, por lo tanto 13 solicitudes están fuera del marco del aborto terapéutico, siendo evaluados bajo el protocolo médico en obstetricia y ginecología del INMP. 
Figura 5. Los expedientes contienen informe de salud mental discutidas en Junta Médica, periodo 2019.
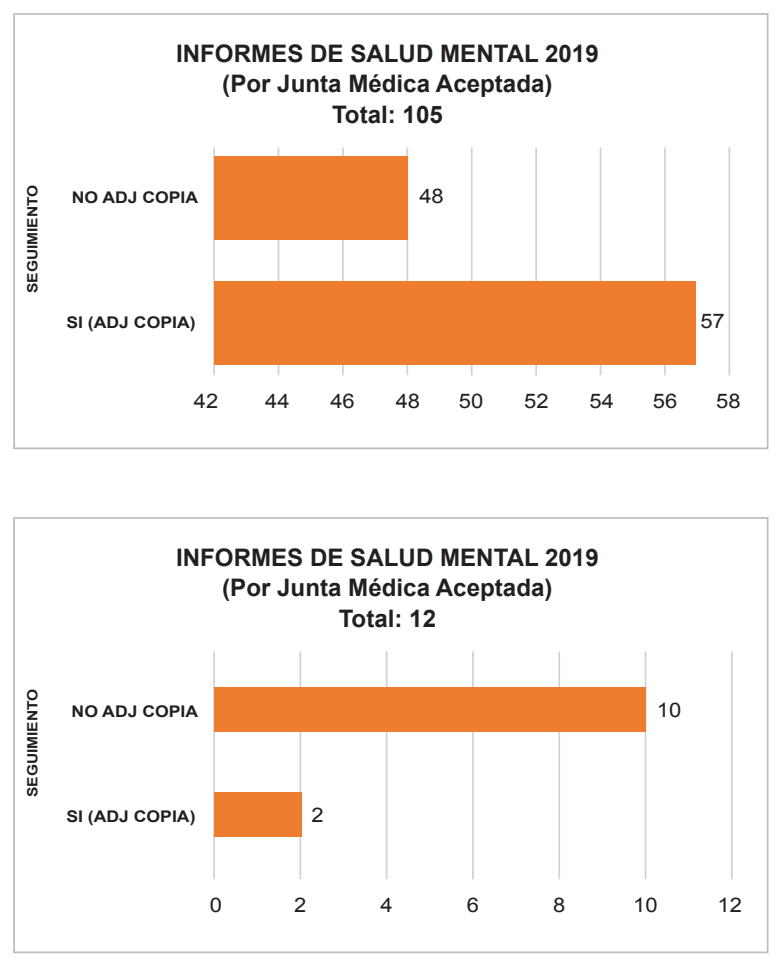

Fuente: Datos obtenidos de los expedientes administrativos que obran en los archivos de la Dirección General del INMP (2021)

Como se aprecia en la figura 5 , de la revisión de las solicitudes de interrupción terapéutica voluntaria del embarazo, en el año 2019, de los 105 casos obran solo en 57 expedientes copia del informe del médico psiquiatra, sin embargo en la junta médica si han señalado en los 48 casos restantes que si cuentan con opinión del psiquiatra, lo cual suponemos obran en la historia clínica de la paciente, pero no está adjunto en el expediente administrativo, al parecer una omisión administrativa.

Figura 6. Los expedientes contienen informe de salud mental discutidas en Junta Médica, periodo 2020.

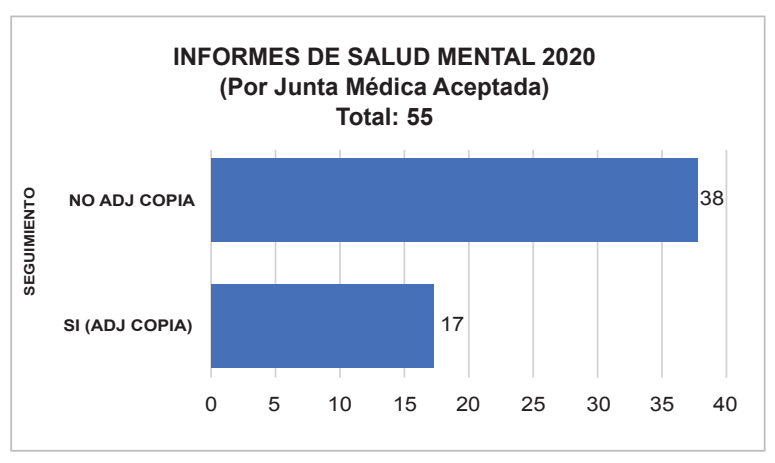

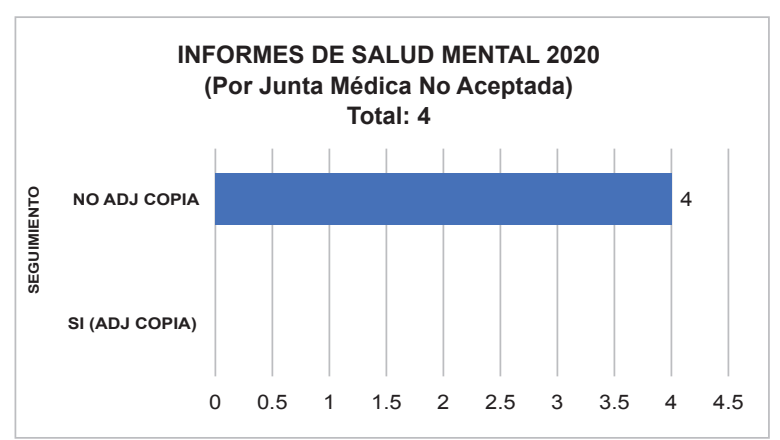

Fuente: Datos obtenidos de los expedientes administrativos que obran en los archivos de la Dirección General del INMP (2021)

Asimismo, como se aprecia en figura 6, además en la revisión de las solicitudes de interrupción terapéutica voluntaria del embarazo, en el año 2020, de los 55 casos obran solo en 17 expedientes copia del informe del médico psiquiatra, sin embargo en la junta médica si han señalado en los 38 casos restantes que si cuentan con opinión del psiquiatra, los cuales obran en la historia clínica de la paciente, pero no está adjunto en el expediente administrativo, al parecer una omisión administrativa, aunado a la pandemia que disminuyó el personal de apoyo administrativo.

\section{DISCUSIÓN}

En líneas generales, se puede observar que todas las solicitudes de interrupción terapéutica voluntaria del embarazo, presentadas por las gestantes, han seguido el procedimiento administrativo para su evaluación mediante junta médica, sin embargo, en algunos expedientes no se ha encontrado copia del informe de salud mental. No obstante, de la lectura de las actas de junta médica se ha considerado la anotación "considerar las recomendaciones de la evaluación especializada de psiquiatría". Por lo tanto, a pesar de no obrar en el expediente la copia de los informes de salud mental, suponemos que ello debe estar consignada en la Historia Clínica de la paciente, en lo cual no alcanza la medición de la presente investigación, pero si consideramos positivo que todos hayan sido evaluados por el profesional médico psiquiatra.

Asimismo, de los resultados de los casos presentados en el Instituto Nacional Materno Perinatal, encontramos que existe relación positiva existe relación positiva entre el derecho a la salud integral y el aborto seguro por indicación terapéutica en contrastación a ello citamos a Marín-Mora AA y col ${ }^{11}$, publicaron un artículo de investigación sobre "La interrupción terapéutica del embarazo como derecho humano a la salud.

Un análisis desde el bioderecho", quienes precisan que la interrupción terapéutica del embarazo o conocido como aborto terapéutico, se ha entendido en diversas 
legislaciones como la interrupción de un embarazo por causas médicas, la que puede motivarse por razones preventivas, como ocurre cuando la gestación pueda empeorar el pronóstico de una enfermedad de base haciendo inviable el feto, o razones curativas, cuando se considera que el embarazo causa un peligro para la vida o la salud física o mental de la gestante. Además sugieren que el debate para construir una argumentación ético-jurídica que logre un consenso sobre la interrupción terapéutica del embarazo, no debe partir únicamente de la contraposición de principios bioéticos en conflicto, o bien de dos derechos humanos fundamentales, entiéndase el Derecho a la vida del embrión o feto, y el derecho a la libre elección o autonomía de la mujer, para decidir su libre desarrollo personal, esta confrontación de dos bienes jurídicos y dos valores constitucionales, no de diferente rango, sino de rango equivalente, debe contemplar además el derecho a la salud o la vida de la mujer, el cual se precisa potencialmente amenazado, lo cual obliga a la intervención médica (previo consentimiento informado) por un denominado estado de necesidad.

Cabe señalar, no obstante, la presente investigación ha sido realizada con la revisión documental de las solicitudes presentadas en el Instituto Nacional Materno Perinatal, por lo que considero que otro aspecto a evaluar debería ser la revisión de la epicrisis en las historias clínicas de cada paciente, a fin de verificar el procedimiento realizado (AMEU o Legrado), lo que corresponde al profesional con especialidad de ginecología y obstetricia.

Se puede concluir, que todas las solicitudes de interrupción terapéutica voluntaria del embarazo, presentadas por las mujeres, han seguido el procedimiento administrativo para su evaluación mediante junta médica, sin embargo, en algunos expedientes no se han encontrado copia del informe de salud mental el cual debe obrar en la historia clínica de cada paciente.

Declaración de conflictos de intereses: La autora declara no tener conflicto de intereses.

Financiamiento: Autofinanciado.

\section{REFERENCIAS BIBLIOGRÁFICAS}

1. Ministerio de Salud. Resolución Ministerial N486-2014/ MINSA, aprueba la Guía Técnica Nacional para la estandarización del procedimiento de la atención integral de la gestante en la interrupción voluntaria por indicación terapéutica del embarazo menor de 22 semanas con consentimiento informado en el marco de lo dispuesto en el artículo $119^{\circ}$ del Código Penal.

2. Constitución Política del Perú (1993), artículo $2^{\circ}$, Toda persona tiene derecho: inciso 1) A la vida, a su identidad, a su integridad moral, psíquica y a su libre desarrollo y bienestar.
3. Constitución Política del Perú (1993), artículo $7^{\circ}$ Derecho a la salud.

4. Código Penal. Decreto Legislativo Nº635 (Lima, 1991), Capítulo II. Aborto, artículo $119^{\circ}$ aborto terapéutico.

5. Ministerio de Salud. Ley 26842, Ley General de Salud y sus modificatorias (Lima, 1997) artículos 3,4 y 5 .

6. Ministerio de Salud. Ley 29414, Ley que establece los Derechos de las Personas Usuarias de los Servicios de Salud (Lima, 2009), modifica el artículo $15^{\circ}$ de la Ley General de la Salud.

7. Távara L, Machare P, García S, Guevara E, Cabrera S, Aguilar J, et al. Barreras para el Aborto Seguro en el Marco de la Ley Peruana. Rev Peru Ginecol Obst.2016; 62(2):153168

8. Colegio Médico del Perú. Código de Ética y Deontología del Colegio Médico del Perú, artículos $53^{\circ}$ y $55^{\circ}$.

9. Instituto Nacional Materno Perinatal. Resolución Directora N 155-2018-DG-INMP, aprueba la guía de práctica clínica y de procedimientos en Obstetricia y Perinatología. Aborto Terapeutico. 2018: 421-433.

10. Rondón MB. Salud mental y aborto terapéutico. An Fac med. 2015; 76(4):407-11. Disponible en: https://revistasinvestigacion.unmsm.edu.pe/index.php/anales/article/ view/11411

11. Marín-Mora AA, Morales-Muñoz K. La interrupción terapéutica del embarazo como derecho humano a la salud. Un análisis desde el bioderecho. Cad. Ibero-Amer. Dir. Sanit. Brasília. 2017; 6(1):167-179. http://dx.doi.org/10.17566/ ciads.v6i1.351

\section{Correspondencia:}

Jacqueline Lourdes Chapa Romero

Dirección: Villa el Salvador, Sector 3, Grupo 11, Mz. M, Lote 15. Villa el Salvador. Lima-Perú.

Correo: jackychapa@gmail.com

Teléfono: 945559545 